\title{
Defining the role of TT-TG and TT-PCL in the diagnosis of lateralization of the Tibial tubercle in recurrent patellar dislocation
}

\author{
Peng Su', Nengri Jian², Beini Mao', Zhong Zhang ${ }^{1}$, Jian $\mathrm{Li}^{1 *}$ and Weili Fu ${ }^{1 *}$
}

\begin{abstract}
Background: The radiological indicators can help doctors determine whether to make tibial tubercle transfer. But which indicator is better is still in question.

Methods: 117 knees in 103 patients who had undergone patellar surgery and 60 knees in 58 patients who had no history of patellar dislocation from 2014 to 2019 were analyzed. Significant differences of tibial tubercle-trochlear groove (TT-TG) on CT and tibial tubercle-posterior cruciate ligament (TT-PCL) on MRI between the case group and the control group were estimated by an unpaired t test. Significant differences between TT-TG on CT and TT-TG on MRI were estimated by a paired t test. The correlation between TT-PCL on MRI and tibial width was estimated by Pearson test. Receiver operating characteristic (ROC) curves and the area under the ROC curve (AUC) were measured to assess the diagnostic accuracy of TT-TG and TT-PCL on MRI.
\end{abstract}

Results: The intraclass correlation coefficient (ICC) for TT-TG between CT and MRI evaluated by two raters was were 0.566. When comparing TT-TG on CT with that on MRI, the mean difference was $2.5 \mathrm{~mm}(p<0.001)$. The mean $T$ TG difference on $C T$ between the case group and the control group was $5.3 \mathrm{~mm}$, which was significantly bigger than the mean TT-PCL difference on MRI of $1.2 \mathrm{~mm}(p<0.001)$. AUC of TT-TG on CT and TT-PCL were 0.838 and 0.580 ( $P<0.001)$. $\Pi$ T-PCL correlated with tibial width $(r=0.450, P<0.001)$.

Conclusion: A statistically significance and a fair ICC proved that TT-TG could not be used interchangeably. The bigger mean difference between the case group and the control group and better AUC proved that TT-TG on CT might be an indicator more suitable for measuring the lateralization of the tibial tubercle. And TT-PCL should be considered as an individual parameter because of the significant correlation between $\Pi T-P C L$ and tibial width.

Keywords: Recurrent patellar dislocation, Computed tomography, Magnetic resonance imaging, Tibial tubercletrochlear groove, Tibial tubercle-posterior cruciate ligament

\footnotetext{
* Correspondence: hxlijian.china@163.com; foxwin2008@163.com

'Department of Orthopaedic Surgery, West China Hospital, Sichuan University, 37 Guoxue lane, Wuhou District, Chengdu, China

Full list of author information is available at the end of the article
}

(c) The Author(s). 2021 Open Access This article is licensed under a Creative Commons Attribution 4.0 International License, which permits use, sharing, adaptation, distribution and reproduction in any medium or format, as long as you give appropriate credit to the original author(s) and the source, provide a link to the Creative Commons licence, and indicate if changes were made. The images or other third party material in this article are included in the article's Creative Commons licence, unless indicated otherwise in a credit line to the material. If material is not included in the article's Creative Commons licence and your intended use is not permitted by statutory regulation or exceeds the permitted use, you will need to obtain permission directly from the copyright holder. To view a copy of this licence, visit http://creativecommons.org/licenses/by/4.0/. The Creative Commons Public Domain Dedication waiver (http://creativecommons.org/publicdomain/zero/1.0/) applies to the data made available in this article, unless otherwise stated in a credit line to the data. 


\section{Background}

Recurrent patellar dislocation is a common disease, especially in adolescents and young adults whose disease rate can be 29 per 100,000 [1]. Many factors can contribute to recurrent patellar dislocation, and surgeons need to select surgical techniques which range from soft tissue surgery to bony correction. Bony procedures include trochleoplasty and medial or distal tubercle transfer. Soft tissue procedures include medial patellofemoral ligament (MPFL) reconstruction and lateral release [2]. For surgeons, whether to do tibial tubercle transfer mainly is determined by the extent of lateralization of tibial tubercle. This is most commonly assessed by TT-TG which is the distance between the anterior tibial tubercle (TT) and the deepest point of the trochlear groove (TG). Goutallier et al. firstly described it on an axial radiograph in 1978 [3]. Subsequently, Dejour measured the TT-TG on CT, making the measurement more precise $[4,5]$.
The cutoff of $20 \mathrm{~mm}$ was considered pathological and as an indication for surgery in recurrent patellar dislocation $[5,6]$. Recently, TT-TG was measured on MRI because of reduced radiation exposure for patients. But The interchangeability of TT-TG between CT and MRI was in controversy. Shoettle et al. argued that the measurement could be used interchangeably between CT and MRI [7]. On the contrary, Anley argued that the measurements for the TT-TG cannot be used interchangeably between CT and MRI. And the cutoff value for TTTG on CT should not be applied to MRI [8].

In recent years, some literatures proved that the measurement of TT-TG could be influenced by the flexion of the knee, For eliminating such effect, Seitlinger et al. proposed a new measurement-TT-PCL which was defined as the distance between the midpoint of the insertion of the patellar tendon and the medial border of the PCL, and recommended $24 \mathrm{~mm}$ as the cutoff value [9].

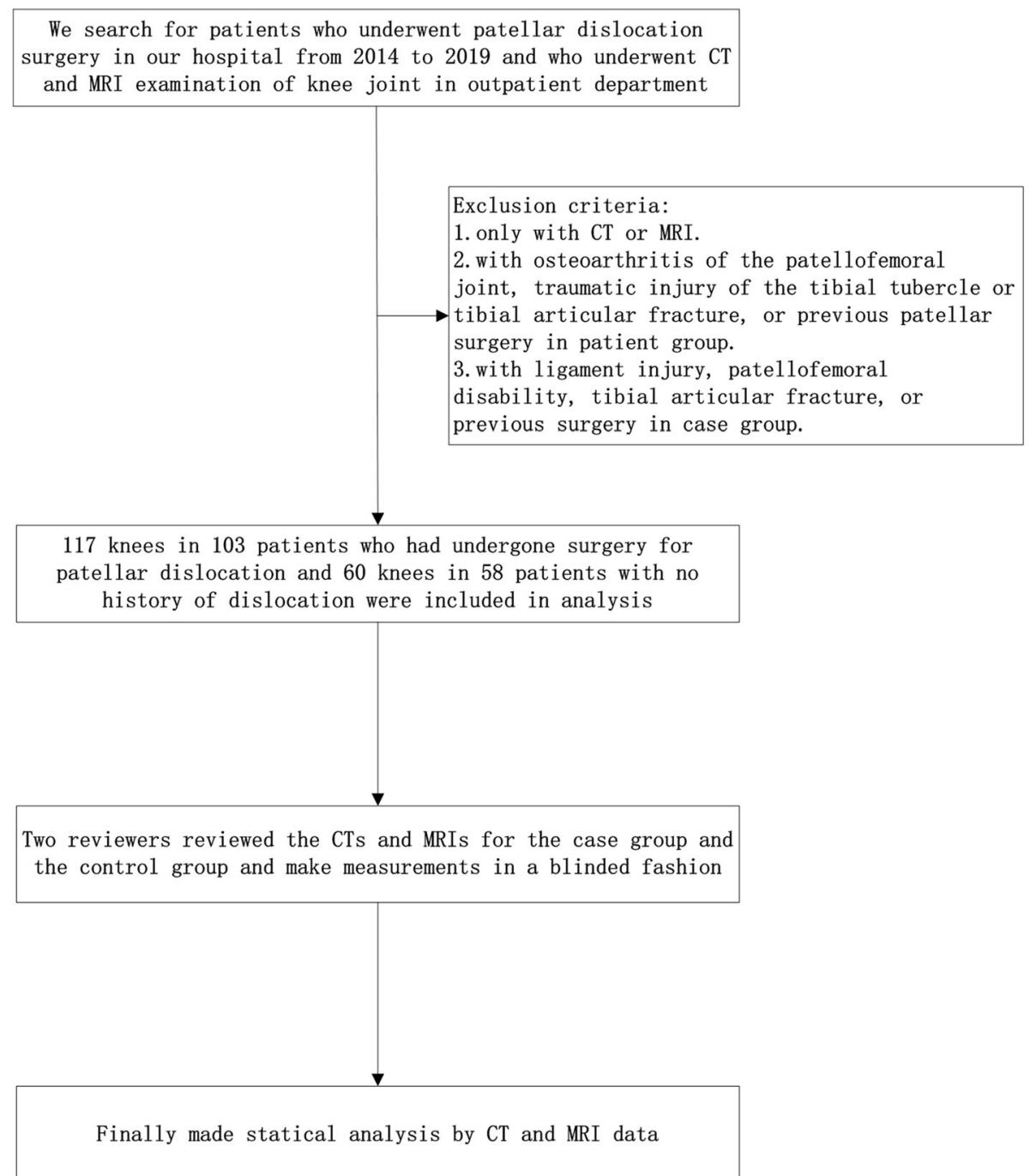

Fig. 1 The flow chart of the research 
But Boutris advocated the use of a new pathologic TTPCL threshold of $21 \mathrm{~mm}$ [10]. Except for TT-PCL, Tensho proposed TT-PCL ratio which was calculated as the TT-PCL distance divided by the tibial width in order to adjust for individual differences [11]. This indicated that the TT-PCL distance might be influenced by the tibial width.

The aim of our study was to determine: 1) TT-TG or TT-PCL, which one was more effective in evaluating patellar displacement. 2) confirm the correlation between the TT-PCL and the width of the tibial plateau.

\section{Methods}

Before the research was started, it was approved by the hospital ethics committee. The imaging data including CT and MRI of 161 patients between 2014 and 2019 was reviewed. The case group consisted of 103 patients (117 knees, age: $19.2 \pm 5$ years old, female/male: 75/28). They all underwent surgery for recurrent patellar dislocation. The main surgical methods were as follows: medial patellofemoral ligament repair or reconstructions, and / or tibial tubercle osteotomy. The patients were excluded in the following situations: osteoarthritis of the patellofemoral joint, traumatic injury of the tibial tubercle, tibial articular fracture, or previous patellar surgery. The control group consisted of 58 patients (60 knees, mean age: $24.3 \pm 6.4$ years old, female/male: $40 / 18$ ). They had normal knees or synovial chondroma or a bone or soft-tissue tumor around the knee joint. The patients were excluded in the following situations: ligament injury, tibial articular fracture, or previous surgery. All the people included in this study had CTs and MRIs. Two reviewers reviewed the imaging data including $\mathrm{CT}$ and MRI, one experienced sports medicine doctor and one experienced musculoskeletal radiologist. All measurements were made in a blind manner. One month later,
30 samples were randomly selected and two observers repeated the measures to calculated the intra-observer reliability. The imaging data data were analyzed by the software (RadiAnt DICOM Viewer, version 5.5.1).The details were seen in Fig. 1.

\section{TT-TG measurement}

The method of TT-TG measurement was similar to that of Schoettle et al. [7] Firstly, a tangent line to the posterior femoral condyles called posterior condylar line was drawn. Secondly, a perpendicular line to the posterior condylar line called trochlear line was drawn through the deepest point of the trochlear groove. Thirdly, the trochlear line was transferred to the axial plane with the middle point of the tibial tuberosity. The TT-TG distance was the distance from the middle point of the tibial tuberosity to the trochlear line. Bony landmarks were also used to measure the TT-TG distance on MRI. The details were seen in Figs. 2a, b and 3a, b.

\section{TT-PCL measurement}

The method of TT-PCL measurement was similar to that of Anley et al. [12] Firstly, a tangent line to the dorsal tibial condyles on the slice just between the articular cartilage and the proximal head of the fibula called the tibial dorsal condylar line was drawn. Secondly, a perpendicular line to the tibial dorsal condylar line called the tibial plateau line was drawn through the medial border of the PCL. Thirdly, the tibial plateau line was transferred to the axial plane with the midpoint of the inferior patellar tendon insertion at the TT. the TT-PCL distance was the distance from the midpoint of the inferior patellar tendon insertion at the TT to the tibial plateau line. The details were seen in Fig. 4a, b.
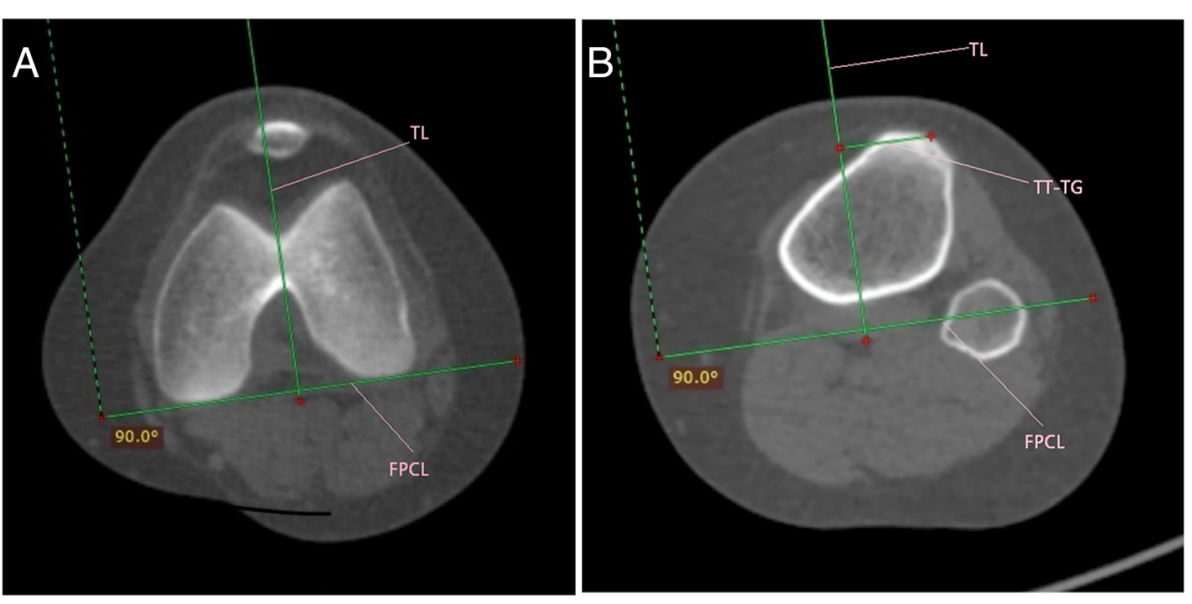

Fig. 2 a, b: Technique for measuring TT-TG distance on Axial computed tomography. $F P C L$, femoral posterior condylar line; $T L$, trochlear line; $T T-$ TG, tibial tubercle-trochlear groove distance 

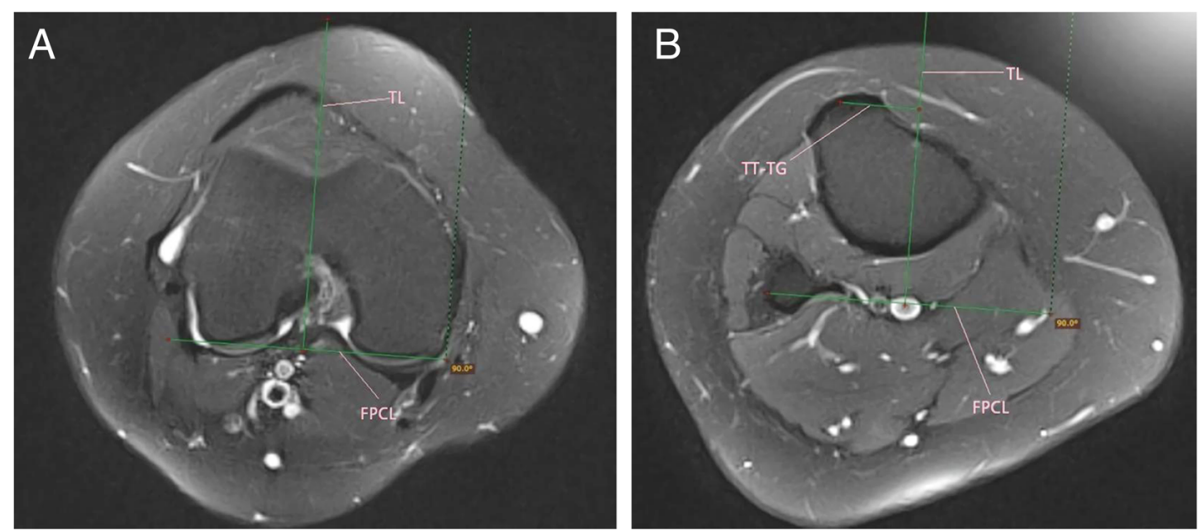

Fig. 3 a, b: Technique for measuring TT-TG distance on Axial magnetic resonance. FPCL, femoral posterior condylar line; TL, trochlear line; TT-TG, tibial tubercle-trochlear groove distance

\section{The tibial width}

The method of tibial width measurement was similar to that of Tensho et al. [11] The measurement was performed on the axial plane where the posterior tibial condyles was clearly recognized. Two perpendicular lines to the tibial posterior condylar line were drawn through the medial and lateral margins of the tibial condyle respectively. The tibial width was the distance between two perpendicular lines. The details were seen in Fig. 5.

\section{CT protocol}

The patient's position was supine with knees straight. A 64-detector row Siemens CT scanner (Siemens Somatom Definition AS+, Germany) was used for examination. The parameters were as follows: matrix $(512 \times$ 512 ), thickness (1-3 mm), scan time (approximately 5 to $10 \mathrm{~s}$ ), 0-mm skip between slices, FOV (20) and bone kernel.

\section{MRI protocol}

The patient's position was supine with knees in a coil. A 1.5-T Siemens MRI scanners (Siemens Magntom Avanto, Germany) with axial T2 imaging in all cases was used for examination. The parameters were as follows: fast spin echo, repetition time (TR) (3000 to 5000 milliseconds), echo time (TE) (40 milliseconds), thickness ( $3 \mathrm{~mm}$ ), scan time (approximately 3-min), 0.6-mm skip between slices, FOV (16), matrix $(352 \times 288)$, number of excitations (NEX) (3), and echo train length (ETL) (8 to 10).

\section{Statistical analysis}

All relevant data were entered in excel and analyzed statistically using IBM SPSS Statics 25 and MedCalc 15.2.2. An unpaired $t$ test was performed to determine significant differences between the different parameters of the case group and the control group. And a paired t test was performed to determine significant differences between TT-TG on CT and TT-TG on MRI. The value of
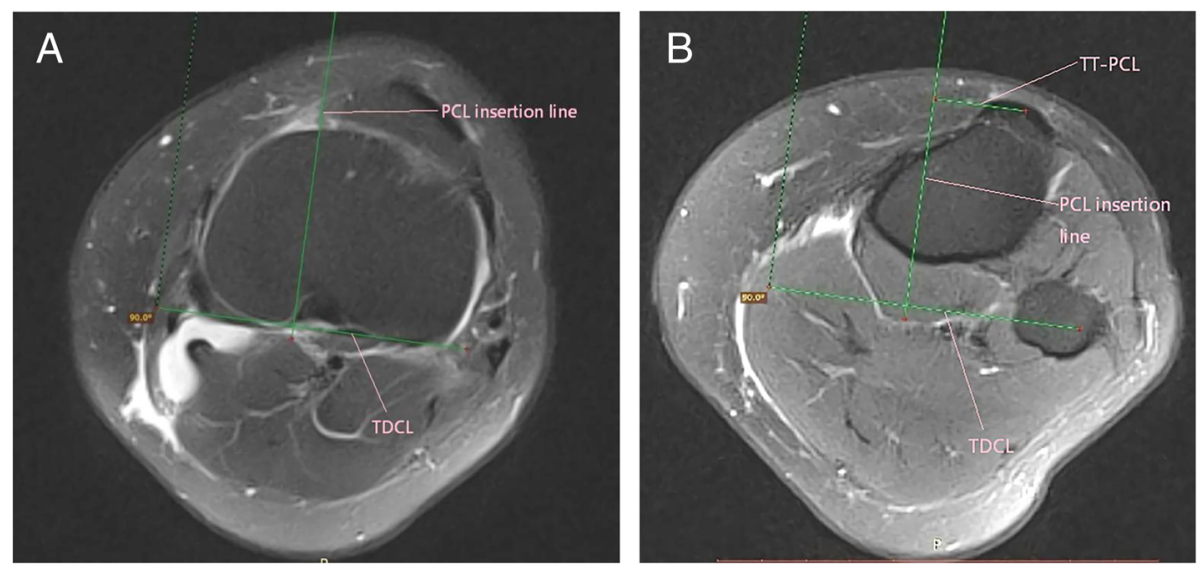

Fig. 4 a, b: Technique for measuring TT-PCL distance on Axial magnetic resonance. TDCL, tibial dorsal condylar line; PCL insertion line, posterior cruciate ligament insertion line; TT-PCL, tibial tubercle-posterior cruciate ligament distance 


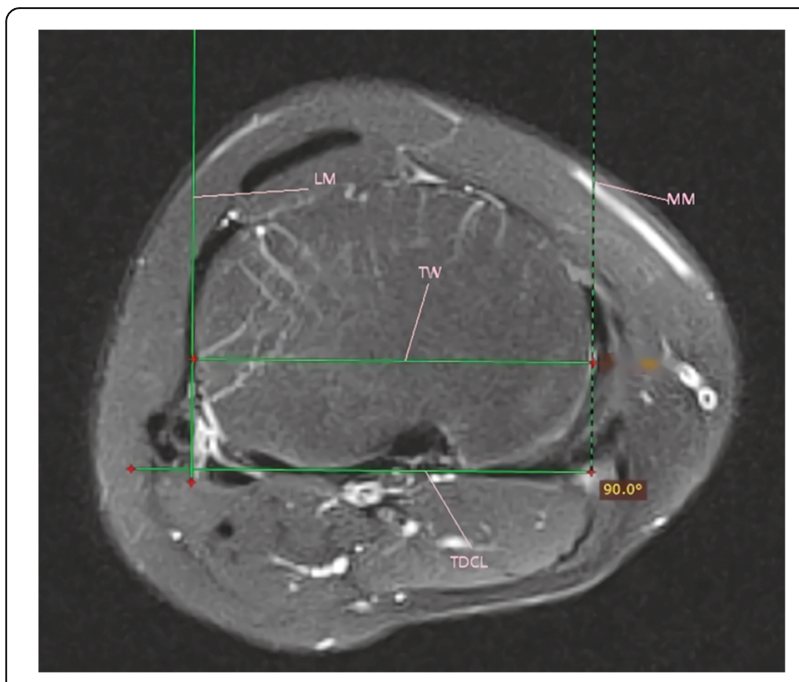

Fig. 5 Technique for measuring tibial width on Axial magnetic resonance. $\mathrm{MM}$, the medial margin of the tibial condyle; LM, lateral margin of the tibial condyle;TW, the distance between the medial and lateral margins of the tibial condyle

$P<0.05$ was considered significant. In addition, the Pearson correlation coefficient was used to calculate the correlation between TT-PCL on MRI and the tibial width. The intraclass correlation coefficient (ICC) and BlandAltman plots was used to evaluate the agreement of multiple measurements by different observers on CT and MRI. An ICC value higher than 0.75 indicates excellent agreement. Receiver operating characteristic (ROC) curves and the area under the ROC curve (AUC) were measured to assess the diagnostic accuracy of different measurements with the cutoffs calculated.

\section{Results}

All patients included in this study had patellar dislocation at least twice, which had a significant negative impact on patients' life. The mean age of the case group in the study was 19 years (range, $12-38$ years). There was a total of 28 male and 75 female patients, and 14 of these patients were with bilateral patellar dislocations. The mean age of the control group was 24 years (range, 1335 years). There was a total of 18 male and 40 female patients, and two of these patients were with bilateral patellar dislocations.
The mean TT-TG distances of the case group on CT and MRI were $22.1 \mathrm{~mm}$ and $19.6 \mathrm{~mm}$, respectively. The mean TT-TG distances of the control group on CT and MRI were $16.8 \mathrm{~mm}$ and $14.3 \mathrm{~mm}$, respectively. The intraclass correlation coefficients (ICC) for TT-TG on CT was excellent (0.974; 95\% CI, 0.964-0.981; $p<0.001)$. The ICC for TT-TG on MRI was excellent $(0.937 ; 95 \%$ CI, 0.900-0.959; $p<0.001)$. The ICC for TT-TG on CT was slightly higher than that on MRI. The mean TTPCL distance of the case group on MRI was $23.0 \mathrm{~mm}$ (range, $14.7-35.1 \mathrm{~mm}$ ). The mean TT-PCL distance of the control group on MRI was $21.8 \mathrm{~mm}$. The ICC for TT-PCL on MRI (0.712; 95\% CI, 0.581-0.798; $p<0.001)$ was worse than that for TT-TG on CT or MRI. The details were seen in Tables 1-2.

Bland-Altman analysis between two raters proved that the mean difference of TT-TG on CT between rater A and $\mathrm{B}$ was $-0.2 \mathrm{~mm}(95 \% \mathrm{CI},-2.3-1.8)$. The mean difference of TT-TG on MRI between rater A and B was $0.7 \mathrm{~mm}$ (95\% CI, -4-2.7). And the mean difference of TT-PCL on MRI between rater A and B was $-1.2 \mathrm{~mm}$ (95\% CI, - 6.7-4.4). The details were seen in Figs. 6, 7 and 8.

When evaluating the variability of TT-TG on the two imaging modalities (CT and MRI), the ICC for TT-TG on the two imaging modalities evaluated by rater A was 0.566 (95\% CI, 0.271-0.731; $p<0.001$ ). The ICC for TTTG on the two imaging modalities evaluated by rater $\mathrm{B}$ was 0.566 (95\% CI, 0.342-0.708; $p<0.001)$. When comparing the TT-TG distance on CT with that on MRI, the mean TT-TG distance between the two imaging modalities was $2.5 \mathrm{~mm}(95 \% \mathrm{CI}, 1.9-3.1 ; p<0.001)$. There was a significant difference on the TT-TG distance between the two imaging modalities, which indicated the TT-TG distance could not be exchangeable between the two imaging modalities.

The mean tibial width on MRI in the case group was $69.1 \mathrm{~mm}$. The mean width of tibial plateau on MRI in the control group was $70.8 \mathrm{~mm}$ (range, 61.4-83.2). There was no significant difference on the tibial width between the case group and the control group $(p=0.058)$. Pearson test proved that TT-PCL had a positive significant correlation with the width of tibial plateau $(R=0.455, p<$ $0.001)$, which indicated TT-PCL should be considered as

Table 1 Comparison of control versus case groups. TT-TG: tibial tuberosity-trochlear groove distance. T-PCL: tibial tuberosityposterior cruciate ligament distance

\begin{tabular}{|c|c|c|c|c|c|c|c|c|c|}
\hline \multirow[t]{2}{*}{ parameter } & \multicolumn{4}{|c|}{ case group } & \multicolumn{4}{|c|}{ control group } & \multirow[t]{2}{*}{$P$ Value } \\
\hline & Mean & SD & Min & $\operatorname{Max}$ & Mean & SD & Min & $\operatorname{Max}$ & \\
\hline TT-TG on CT & 22.085 & 3.662 & 13.35 & 31.25 & 16.763 & 4.223 & 4.9 & 30.05 & $<0.0001$ \\
\hline TT-TG on MRI & 19.606 & 4.426 & 5.55 & 33 & 14.255 & 4.466 & 4.95 & 24.75 & $<0.0001$ \\
\hline TT-PCL on MRI & 23.035 & 3.846 & 14.7 & 35.1 & 21.843 & 3.25 & 14.75 & 28.2 & 0.041 \\
\hline Width of tibial plateau & 69.134 & 4.401 & 61.15 & 82.55 & 70.769 & 5.808 & 61.35 & 83.2 & 0.058 \\
\hline
\end{tabular}


Table 2 Results of Intraclass Reliability Calculations. ICC: intraclass correlation coefficient; Interrater Reliability: Reliability between rater $A$ and $B$ for a given imaging modality. Intermethod Reliability: Reliability between $\mathrm{CT}$ and MRI for the raters

\begin{tabular}{ll}
\hline Reliability & ICC \\
\hline Interrater & \\
TT-TG on CT & 0.974 \\
TT-TG on MRI & 0.937 \\
TT-PCL on MRI & 0.712 \\
Inter-method & \\
Rater A & 0.566 \\
Rater B & 0.566 \\
\hline
\end{tabular}

an individual parameter in recurrent patellar dislocations.

For the TT-TG distance on CT, the AUC was 0.838 . The sensitivity and specificity were 0.68 and 0.9 , respectively. For the TT-TG distance on MRI, the AUC was 0.814 . The sensitivity and specificity were 0.9 and 0.62 , respectively. For the TT-PCL distance on MRI, the AUC was 0.58 . The sensitivity and specificity were 0.45 and 0.7, respectively. The difference of AUC between TT-TG on CT and TT-PCL on MRI was 0.258 (95\% CI, 0.175$0.341 ; p<0.001)$, which indicated that the diagnostic accuracy of TT-TG on CT was better than that of TT-PCL on MRI. The difference of AUC between TT-TG on MRI and TT-PCL on MRI was 0.234 (95\% CI, 0.140$0.328 ; p<0.001)$, which indicated that the diagnostic accuracy of TT-TG on MRI was better than that of TTPCL on MRI. The details of AUC were seen in Table 3 and Fig. 9.

\section{Discussion}

Firstly, a statistically significant difference of the mean TT-TG difference $(2.5 \mathrm{~mm})$ and only a fair ICC (0.566) for TT-TG between CT and MRI-proved that TT-TG could not be used interchangeably between the two imaging modalities. Secondly, inter-observer reliability for the TT-PCL measurement ( $\mathrm{ICC}=0.712$ ) was worse than that for the TT-TG on CT (ICC=0.914). The mean TTTG difference between the case group and the control group on CT was $5.3 \mathrm{~mm}$, which was obviously bigger than the mean TT-PCL difference of $1.2 \mathrm{~mm}$. No doubt that the increase of the distance difference between the case group and the control group was helpful for doctors to distinguish the patients from the normal. Thirdly, Receiver operating characteristic (ROC) curves and the area under the ROC curve (AUC) were measured to assess the diagnostic accuracy of TT-TG and TT-PCL on MRI. The results proved that the diagnostic accuracy of TT-TG on CT (AUC=0.838) were better that of TTPCL on MRI (AUC=0.58). At last, Pearson test was established to prove that there was a positive correlation

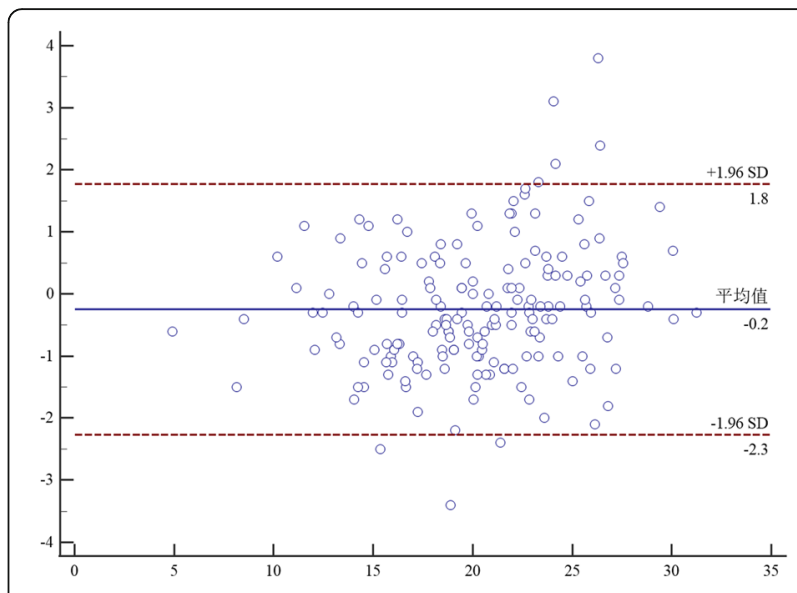

Fig. 6 Bland-Altman analysis of interrater agreement between rater $A$ and $B$ for TT-TG on $C T$

between the TT-PCL distance and the width of tibial plateau $(R=0.455, p<0.001)$.

A few of studies had reported the ICCs for TT-TG on CT and TT-PCL on MRI. Seitlinger et al. [9] noted that the ICC for TT-PCL was 0.74, which was similar with that obtained in our research $(\mathrm{ICC}=0.712)$. Daynes et al. [13] noted the ICC for TT-TG on CT was 0.89 , which was similar with that obtained in our research (ICC= 0.974). From the points above, we can find that the measurements of the TT-TG distance on CT and the TTPCL distance on MRI were reliable, and the reliability of the TT-TG distance on CT was better than the TT-PCL distance on MRI.

In terms of the reliability of the two imaging modalities for TT-TG, Camp et al. [14] noted that the ICCs for TT-TG between two imaging modalities were 0.532 for rater $\mathrm{A}$ and 0.539 for rater $\mathrm{B}$, respectively. In addition, they found that the TT-TG distance on CT was greater than that on MRI with the mean difference of $2.23 \mathrm{~mm}$. Anley et al. [12] noted that the ICCs for TT-TG between two imaging modalities were 0.54 for rater $\mathrm{A}$ and 0.48 for rater $\mathrm{B}$, respectively. In addition, they found the TT-TG distance on CT was greater than that on MRI with the mean difference of $4.16 \mathrm{~mm}$. The results mentioned above were similar to ours. In our research, the ICCs for TT-TG between two imaging modalities were 0.566 for rater $\mathrm{A}$ and 0.566 for rater $\mathrm{B}$, respectively. And the TT-TG distance on CT was greater than that on MRI with the mean difference of $2.5 \mathrm{~mm}$ $(p<0.0001)$. Considering the low ICC and the significant difference of TT-TG distance between 2 imaging modalities, TT-TG distance between two imaging modalities could not be interchangeable. For the lower values for TT-TG on MRI, it might be caused by increased flexion of the knee with the use of a MRI knee coil [12].

In terms of TT-PCL, Boutris et al. [10] noted that the mean TT-PCL distance of the case group and the 


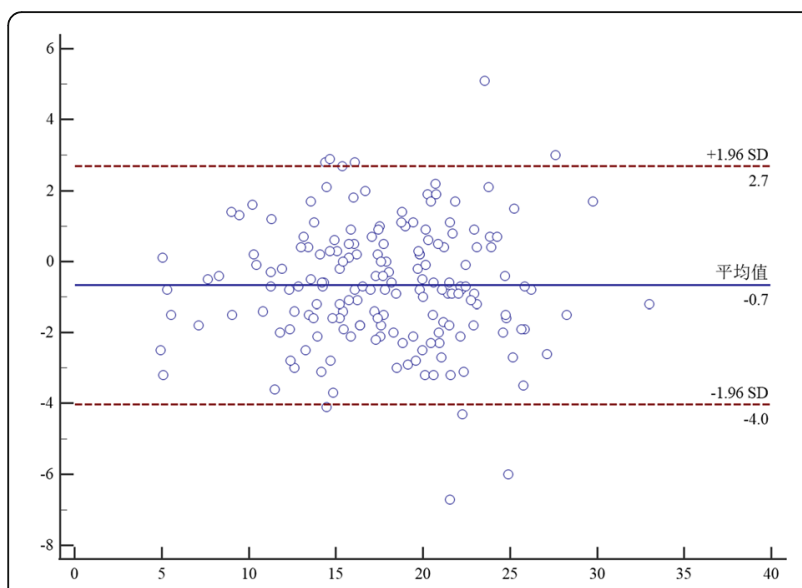

Fig. 7 Bland-Altman analysis of interrater agreement between rater $A$ and $B$ for TT-TG on MRI

control group was $21.1 \pm 4.1$ and $18.8 \pm 4.0 \mathrm{~mm}$, respectively. Daynes et al. [13] noted that the mean TTPCL of the case group and the control group was 21.62 \pm 4.52 and $19.04 \pm 4.51 \mathrm{~mm}$, respectively. In terms of TT-TG on CT, Tensho et al. [11] noted that the mean TT-TG distance of the case group and the control group was $19.2 \pm 4.0$ and $14.3 \pm 2.9 \mathrm{~mm}$, respectively. Dejour et al. [5] noted that the mean TT-TG distance of the case group and the control group was $19.8 \pm 1.6$ and $12.7 \pm 3.4 \mathrm{~mm}$, respectively. In our research, the mean TT-TG distance of the case group and the control group was $22.1 \pm 3.7$ and $16.8 \pm 4.2 \mathrm{~mm}$. The mean TT-PCL distance of the case and control group was $23 \pm 3.8$ and $21.8 \pm 3.3 \mathrm{~mm}$. Obviously, the mean TT-TG difference on CT between the case group and the control group was greater than the mean TT-PCL difference, which indicated TT-TG on CT more helpful for doctors to differentiate between patients and the normal.

In addition, Receiver operating characteristic (ROC) curves and the area under the ROC curve (AUC) were established to assess the diagnostic accuracy of TT-TG and TT-PCL. The AUCs of TT-TG on CT and TT-PCL on MRI were 0.838 and 0.58 , respectively, which were approximate to those obtained by Tensho et al. (0.84 and 0.66 , respectively). It was concluded from the above that the diagnostic accuracy of TT-TG on CT was better than that of TT-PCL on MRI. However, when trochlear

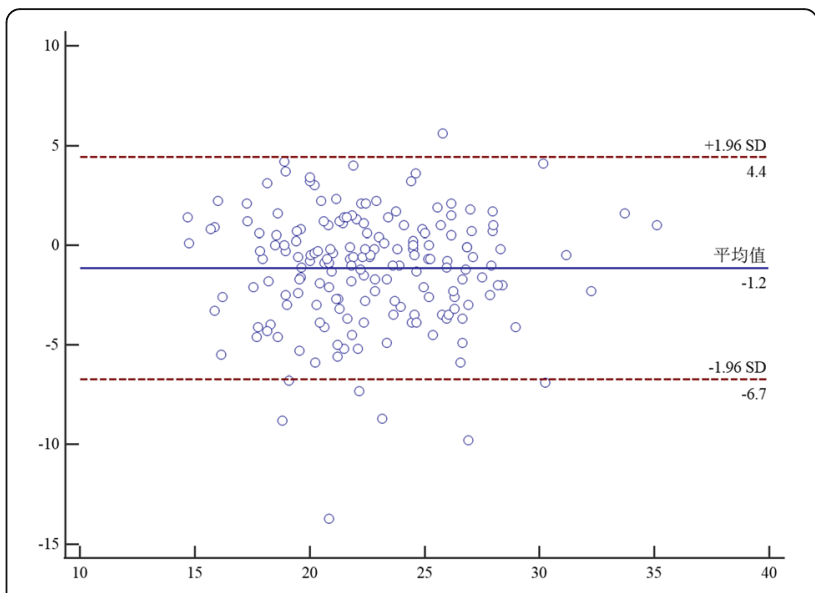

Fig. 8 Bland-Altman analysis of interrater agreement between rater $A$ and $B$ for TT-PCL on MRI

dysplasia existed, it was difficult to determine the deepest point of tibial tubercle-trochlear groove. In such a situation, TT-PCL was an ideal choice.

Considering the individual difference, TT-PCL ratio was proposed by Tensho et al. [11] However, few articles proved the correlation between the TT-PCL distance on MRI and the tibial width [15]. In this research, Pearson test was established to confirm the correlation between the TT-PCL distance on MRI and the tibial width. The results indicated that TT-PCL had a positive significant correlation with the width of tibial plateau $(R=0.455, p<$ 0.001). Considering this factor, TT-TG should be considered as an individual parameter in recurrent patellar dislocations though it is not affected by the flexion of the knees.

The cases included in this study all underwent surgery for patellar dislocation, while the cases in the other studies were described with dislocation more than twice, without illustrating the frequency of dislocation in detail. In clinical setting, the patients choose the surgery as the therapy, always because dislocation so frequently affects the normal life. And TT-TG>20 mm was considered as a standard to make the transferring of tibial tubercle. The patients who underwent surgery for patellar dislocation might be more suitable for the scientific research. This article is limited by the retrospective nature. The position of the knee joint may affect the results of our research.

Table 3 Diagnostic Performance Parameters of the Measurements

\begin{tabular}{lllllll}
\hline Parameter & AUC & $\mathbf{9 5 \% ~ C l}$ & $\boldsymbol{P}$ Value & Cutoff & Sensitivity & Specificity \\
\hline TT-TG on CT & 0.838 & $0.775-0.889$ & $<0.0001$ & 20.6 & 0.68 & 0.9 \\
TT-TG on MRI & 0.814 & $0.748-0.868$ & $<0.0001$ & 14.7 & 0.9 & 0.62 \\
TT-PCL on MRI & 0.58 & $0.503-0.653$ & 0.0744 & 23.3 & 0.45 & 0.7 \\
\hline
\end{tabular}




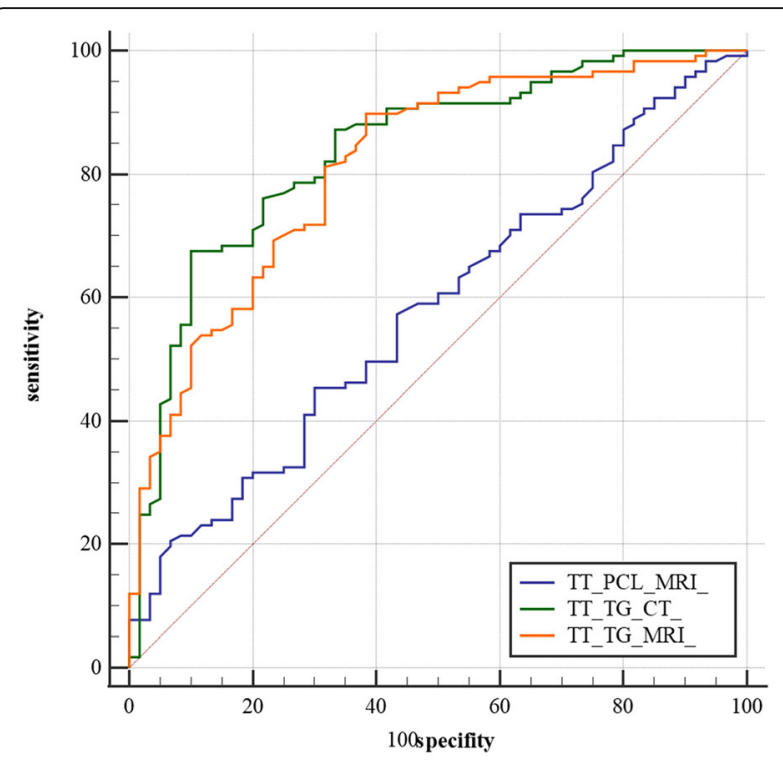

Fig. 9 ROC curve showing the AUCS of the TT-TG distance on $C T$, TT-TG distance on MRI and TT-PCL distance on MRI

\section{Conclusion}

A statistically significance and a fair ICC proved that TT-TG could not be used interchangeably between the two imaging modalities. The bigger mean difference between the case group and the control group and better AUC proved that TT-TG on CT might be an indicator more suitable for measuring the lateralization of the tibial tubercle. And TT-PCL should be considered as an individual parameter because of the significant correlation with tibial width.

\section{Abbreviations}

RPD: Recurrent patellar dislocation; CT: Computed tomography;

MRI: Magnetic resonance imaging; TT-TG: Tibial tubercle-trochlear groove; TT-PCL: Tibial tubercle-posterior cruciate ligament; ICC: Intraclass correlation coefficient; ROC: Receiver operating characteristic; AUC: Area under the ROC curve; MPFL: Medial patellofemoral ligament

\section{Acknowledgements}

The authors would like to thank KB Zhang SK Lai for their participation in the data collection process of this study.

\section{Conflict of interests}

Each author certifies that he or she has no commercial associations (eg, consultancies, stock ownership, equity interest, patent/licensing arrangements, etc) that might pose a conflict of interest in connection with the article.

\section{Authors' contributions}

NJ ZZ and BM participated in the data collection, and analysis. PS WF and JL contributed to the study design and conception, and revision of the manuscript. All authors read and approved the final manuscript.

\section{Availability of data and materials}

The datasets supporting the conclusions of this article are included within the article. Raw data can be requested from the corresponding author.

\section{Ethics approval and consent to participate}

The protocol for the study was approved by Ethics Committee on Biomedical Research of West China Hospital of Sichuan University that all investigations were conducted in conformity with ethical principles. This study met the conditions for exemption of informed consent and was approved by Ethics Committee on Biomedical Research. No administrative permissions were required to access the raw data used in this study. The data used in this study was anonymised before its use.

\section{Consent for publication}

Not applicable.

\section{Competing interests}

The authors declare that they have no financial or non-financial competing interests.

\section{Author details}

1Department of Orthopaedic Surgery, West China Hospital, Sichuan University, 37 Guoxue lane, Wuhou District, Chengdu, China. ${ }^{2}$ Department of Radiology, West China Hospital, Sichuan University, 37 Guoxue lane, Wuhou District, Chengdu, China.

Received: 4 September 2020 Accepted: 21 December 2020 Published online: 08 January 2021

\section{References}

1. Fithian DC, Paxton EW, Stone ML, Silva P, Davis DK, Elias DA, White LM. Epidemiology and natural history of acute patellar dislocation. Am J Sports Med. 2004;32(5):1114-21.

2. Weber AE, Nathani A, Dines JS, Allen AA, Shubin-Stein BE, Arendt EA, Bedi A. An algorithmic approach to the Management of Recurrent Lateral Patellar Dislocation. JBJS. 2016;98(5):417-27.

3. Goutallier D, Bernageau J, Lecudonnec B. The measurement of the tibial tuberosity. Patella groove distanced technique and results (author's transl). Rev Chir Orthop Reparatrice Appar Mot. 1978;64(5):423-8.

4. Wagenaar FC, Koeter S, Anderson PG, Wymenga AB. Conventional radiography cannot replace $C T$ scanning in detecting tibial tubercle lateralisation. Knee. 2007;14(1):51-4.

5. Dejour H, Walch $G$, Nove-Josserand L, Guier C. Factors of patellar instability: an anatomic radiographic study. Knee Surg Sports Traumatol Arthrosc. 1994; 2(1):19-26.

6. Sherman SL, Erickson BJ, Cvetanovich GL, Chalmers PN, Farr J 2nd, Bach BR $\mathrm{Jr}$, Cole BJ. Tibial tuberosity osteotomy: indications, techniques, and outcomes. Am J Sports Med. 2014;42(8):2006-17.

7. Schoettle PB, Zanetti M, Seifert B, Pfirrmann CW, Fucentese SF, Romero J. The tibial tuberosity-trochlear groove distance; a comparative study between CT and MRI scanning. Knee. 2006;13(1):26-31.

8. Steensen RN, Bentley JC, Trinh TQ, Backes JR, Wiltfong RE. The prevalence and combined prevalences of anatomic factors associated with recurrent patellar dislocation: a magnetic resonance imaging study. Am J Sports Med. 2015;43(4):921-7.

9. Seitlinger G, Scheurecker G, Hogler R, Labey L, Innocenti B, Hofmann S. Tibial tubercle-posterior cruciate ligament distance: a new measurement to define the position of the tibial tubercle in patients with patellar dislocation. Am J Sports Med. 2012:40(5):1119-25.

10. Boutris N, Delgado DA, Labis JS, McCulloch PC, Lintner DM, Harris JD. Current evidence advocates use of a new pathologic tibial tubercleposterior cruciate ligament distance threshold in patients with patellar instability. Knee Surg Sports Traumatol Arthrosc. 2018;26(9):2733-42.

11. Tensho K, Shimodaira H, Akaoka Y, Koyama S, Hatanaka D, Ikegami S, Kato $\mathrm{H}$, Saito N. Lateralization of the Tibial tubercle in recurrent patellar dislocation: verification using multiple methods to evaluate the Tibial tubercle. J Bone Joint Surg Am. 2018;100(9):e58.

12. Anley CM, Morris GV, Saithna A, James SL, Snow M. Defining the role of the Tibial tubercle-trochlear groove and Tibial tubercle-posterior cruciate ligament distances in the work-up of patients with Patellofemoral disorders. Am J Sports Med. 2015;43(6):1348-53.

\section{Funding}

No funding. 
13. Daynes J, Hinckel BB, Farr J. Tibial tuberosity-posterior cruciate ligament distance. J Knee Surg. 2016;29(6):471-7.

14. Camp CL, Stuart MJ, Krych AJ, Levy BA, Bond JR, Collins MS, Dahm DL. CT and MRI measurements of tibial tubercle-trochlear groove distances are not equivalent in patients with patellar instability. Am J Sports Med. 2013;41(8): 1835-40.

15. Pozzi G, Al-Mnayyis A, Almolla J, Albano D, Messina C, Merli I, Mesquita RD, Sconfienza LM. Middle patellar tendon to posterior cruciate ligament (PTPCL) and normalized PT-PCL: new magnetic resonance indices for tibial tubercle position in patients with patellar instability. Knee. 2018;25(5):799806.

\section{Publisher's Note}

Springer Nature remains neutral with regard to jurisdictional claims in published maps and institutional affiliations.

Ready to submit your research? Choose BMC and benefit from:

- fast, convenient online submission

- thorough peer review by experienced researchers in your field

- rapid publication on acceptance

- support for research data, including large and complex data types

- gold Open Access which fosters wider collaboration and increased citations

- maximum visibility for your research: over $100 \mathrm{M}$ website views per year

At BMC, research is always in progress.

Learn more biomedcentral.com/submissions 